\section{Acute obstructive hydrocephalus after subarachnoid haemorrhage}

Neurological deterioration within the first week after subarachnoid haemorrhage may be due to a haematoma, rebleed, or vasospasm and ischaemia. Although communicating hydrocephalus occurring within the first week may also contribute to deterioration, ${ }^{1-3}$ only rarely has acute obstructive (non-communicating) hydrocephalus been reported as a cause. ${ }^{4}$ We describe two cases in which this complication occurred within 12 hours after subarachnoid haemorrhage.

\section{Case reports}

Case 1-A 64 year old woman was admitted to a medical ward a few hours after sudden onset of severe headache associated with photophobia and neck stiffness. She was orientated and had no neurological deficit. Subarachnoid haemorrhage was confirmed by lumbar puncture. Two hours after admission her level of consciousness deteriorated slightly and she was subsequently transferred to our neurosurgical unit. On admission she was able to localise to a painful stimulus but did not open her eyes to speech and gave no verbal response. Emergency CT scan showed an acute obstructive hydrocephalus due to blood within the third ventricle (see figure). Five hours after the episode of subarachnoid haemorrhage a ventricular drain was inserted through a right frontal burr hole. Four hours later her level of consciousness had improved; she obeyed commands, gave a confused verbal response, and opened her eyes to speech. She later required a ventriculoperitoneal shunt.

Case 2-A 54 year old woman was awakened by severe occipital headache and vomiting. Later that day she was admitted to a general surgical ward, at which time she was obeying commands, talking, and opening her eyes to
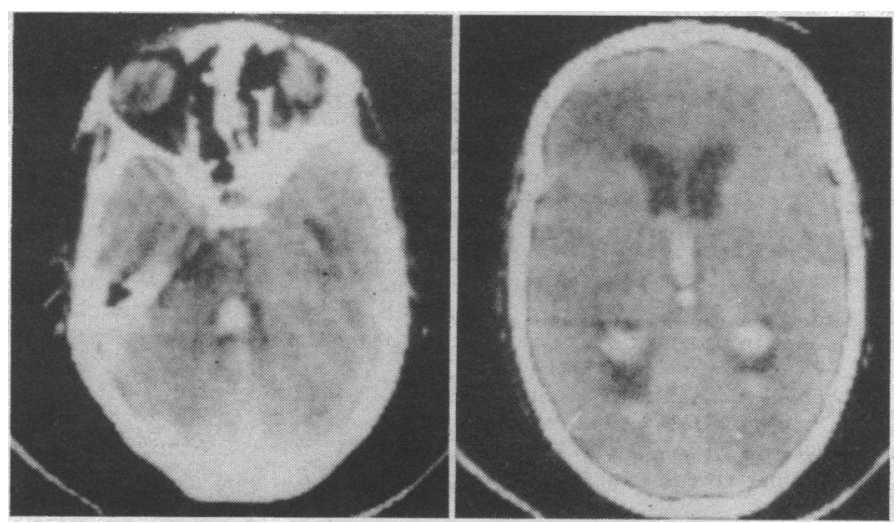

Case 2. CT scan of patient with acute obstructive hydrocephalus showing blood in all four ventricles.

speech. Lumbar puncture confirmed a subarachnoid haemorrhage. Her level of consciousness gradually deteriorated over eight hours and she was transferred to our unit. On admission she was able to localise to a painful stimulus but did not open her eyes to speech and gave no verbal response. A CT scan 12 hours after the initial episode showed an acute obstructive hydrocephalus with blood in all four ventricles (figure). Bifrontal ventricular drains were immediately inserted, and within two hours her level of consciousness had improved to obeying commands, giving a confused verbal response, and opening eyes to speech.

\section{Comment}

Sudden neurological deterioration in the first week after a subarachnoid haemorrhage may be due to either a rebleed or raised intracranial pressure caused by an expanding haematoma or associated brain swelling. Neurological deterioration progressing more slowly may be due to delayed ischaemia or development of communicating hydrocephalus. We describe acute obstructive hydrocephalus as a further cause of deterioration in two patients. Obstructive hydrocephalus is a reasonable explanation for the deterioration in consciousness in these patients because they improved soon after ventricular drainage. The role of the CT scan in the diagnosis and management of subarachnoid haemorrhage is well established. ${ }^{5}$ How common this readily treatable cause of progressive neurological deterioration is in subarachnoid haemorrhage is unknown. Increased use of the CT scan in the primary diagnosis of subarachnoid haemorrhage is likely to provide further information. Ventricular drainage may contribute to the early management of this condition.

We thank Mr T A H Hide for permission to report on his patients.

${ }^{1}$ Raimondi AJ, Torres H. Acute hydrocephalus as a complication of subarachnoid hemorrhage. Surg Neurol 1973;1:23-6.

2 Vassilouthis J, Richardson AE. Ventricular dilatation and communicating hydrocephalus following spontaneous subarachnoid hemorrhage. $\mathfrak{f}$ Neurosurg 1979;51:341-51.

${ }^{3}$ Doczi T, Menessanji Z, Szegvary Z, Huszka E. Disturbances of cerebrospinal fluid circulation during the acute stage of subarachnoid hemorrhage. Neurosurgery 1983;12:435-8.

Kusske JA, Turner PR, Ojemann GA, Harris AB. Ventriculostomy for the treatment of acute hydrocephalus following subarachnoid hemorrhage. f Neurosurg 1975;38:591-5.

5 Kendell BE, Claveria E. Computerised tomography in subarachnoid haemorrhage. Br $\mathcal{F}$ Radiol 1976;49:483-501.

(Accepted 25 fanuary 1984)

Institute of Neurological Sciences, Southern General Hospital, Glasgow G51 4TF

Y M KOHI, FRCS, senior registrar

R A JOHNSTON, MD, FRCS, senior registrar

U P DEVKOTA, MB, BS, registrar

Correspondence to: Mr Y M Kohi.

\section{Response of urinary albumin to submaximal exercise in newly diagnosed non-insulin dependent diabetes}

Subtle changes in urinary albumin excretion are the first clinical indication of renal disease in diabetes mellitus and are thought to be caused by the excessive glycosylation of glomerular proteins. Exercise increases pathological albuminuria, and the response to standardised submaximum exercise provides a highly sensitive test of early glomerular dysfunction in diabetes. ${ }^{1}$ Thus albumin excretion remains unchanged in healthy people but may rise severalfold in clinically established insulin dependent diabetes. ${ }^{12}$ Screening studies suggest that non-insulin dependent diabetes is often preceded by a long period of asymptomatic hyperglycaemia, and the aim of our investigation in patients with newly diagnosed non-insulin dependent diabetes was to establish whether renal dysfunction was already established by the time the diabetes declared itself clinically.

\section{Patients, methods, and results}

The albuminuric response to submaximal exercise was examined in 10 newly diagnosed and untreated patients with non-insulin dependent diabetes (six men and four women aged 45-70) and seven healthy controls. A diuresis was first established by having the subjects consume $250 \mathrm{ml}$ of tap water every 20 minutes for 90 minutes before and during the test. Urine was collected over periods of 20 minutes immediately before and five minutes after a 10 minute exercise period on a treadmill. The heart rate on the treadmill was maintained between 100 and 130 beats/min in the men and $130-165$ beats/ $\mathrm{min}$ in the women, equivalent to the $600 \mathrm{kpm} / \mathrm{min}$ bicycle stress test used by Mogensen and Vittinghus when studying albuminuria in insulin dependent diabetes. ${ }^{1}$ The test was performed before treatment and repeated four and eight weeks after the introduction of a high fibre, energy controlled diet.

Albumin was measured by a single sandwich enzyme linked immunosorbent assay with a coefficient of variation of less than $10 \%$ (Mohamed $A$, unpublished study). Capillary glucose was measured by BM Stix (Boehringer) 